Recepción: 20 / 04 / 2017

Aceptación: 20 / 05 / 2017

Publicación: 15 / 07 / 2017

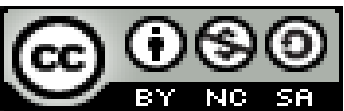

Ciencias de la Educación

Artículo Científico

\title{
La lectura comprensiva en el desarrollo intelectual de los niños y niñas en la educación general básica media
}

Comprehensive reading in the intellectual development of boys and girls in basic general basic education

\section{A compreensão da leitura no desenvolvimento intelectual das crianças no ensino geral básico e secundário}

\author{
Viviana P. Patiño-Zambrano ${ }^{\text {I }}$ \\ viviana.patinoz@ug.edu.ec \\ Alexandra M. Alvarado-Álvarez ${ }^{\mathrm{II}}$ \\ alexandra.alvaradoa@ug.edu.ec \\ María D. Zambrano-Mendoza III \\ mariad.zambranom@educacion.gob.ec \\ José A. Barberan-Zambrano IV \\ arturo.barberan@educacion.gob.ec
}

Correspondencia: viviana.patinoz@ug.edu.ec

\footnotetext{
Magister en Gerencia de Salud para el desarrollo local; Universidad de Guayaquil, Guayaquil, Ecuador. Magister en Gerencia de Servicios de Salud; Universidad de Guayaquil, Guayaquil, Ecuador. Docente; Ministerio de Educación del Ecuador, Universidad de Guayaquil, Guayaquil, Ecuador. Docente; Ministerio de Educación del Ecuador, Universidad de Guayaquil, Guayaquil, Ecuador.
} 
Viviana P. Patiño-Zambrano; Alexandra M. Alvarado-Álvarez; María D. Zambrano-Mendoza; José A. Barberan-Zambrano

\section{Resumen}

Esta investigación es de gran interés porque la lectura es uno de los instrumentos más poderosos de aprendizaje. Quien lee adecuadamente puede utilizar lo leído para ampliar sus pensamientos y reflexionar sobre lo escrito. Así, la lectura se convierte en una herramienta que permite pensar y aprender. La práctica docente la presupone como uno de los medios de adquisición e interpretación de conocimientos, de informaciones, de experiencias; vale decir, como una forma de aprendizaje que permite adquirir otros aprendizajes.

Es de mucha importancia conceptualizar a la lectura como una fuente de información, de aprendizaje, que permite resolver problemas tanto del contexto escolar como de la vida cotidiana, posibilitando ampliar la visión del mundo, el desarrollo de la sensibilidad y los procesos intelectuales, desde esta perspectiva, esta investigación es importante, pues posibilita sintetizar nuevos conocimientos a partir de informaciones previas; de igual manera, a través de ella se conocieron y comprendieron los conocimientos producidos en diversos tiempos y en diferentes temáticas.

El objetivo es determinar el efecto de la lectura comprensiva en el desarrollo intelectual de los niños y niñas de quinto grado en la Educación General Básica Media de la Escuela 28 de mayo de la Parroquia Canuto Cantón Chone período 2014 - 2015.

Palabras claves: Lectura compresiva; desarrollo intelectual; docente; estudiantes y padres. 


\section{Abstract}

This research is of great interest because reading is one of the most powerful learning tools. Whoever reads properly can use what has been read to broaden their thoughts and reflect on what is written. Thus, reading becomes a tool that allows thinking and learning. The teaching practice presupposes it as one of the means of acquiring and interpreting knowledge, of information, of experiences; That is to say, as a form of learning that allows to acquire other learnings.

It is very important to conceptualize reading as a source of information, of learning, that allows solving problems both in the school context and in daily life, making it possible to expand the world view, the development of the sensibility and the intellectual processes, from this Perspective, this research is important, since it makes it possible to synthesize new knowledge from previous information; In the same way, through it the knowledge produced in different times and in different themes was known and understood.

The aim is to determine the effect of comprehensive reading on the intellectual development of fifth graders in the Basic General Basic Education of the May 28th School of Canuto Parish Canton Chone from 2014 to 2015.

Key words: Compressive reading; intellectual development; teacher; students and parents. 
Viviana P. Patiño-Zambrano; Alexandra M. Alvarado-Álvarez; María D. Zambrano-Mendoza; José A. Barberan-Zambrano

\section{Resumo}

Esta pesquisa é de grande interesse porque a leitura é uma das ferramentas de aprendizagem mais poderosos. Quem lê ler corretamente pode usá-lo para expandir seus pensamentos e refletir sobre a escrita. Assim, a leitura se torna uma ferramenta para pensar e aprender. prática de ensino pressupõe como um dos meios de aquisição e interpretação do conhecimento, informação, experiência; ou seja, como uma forma de aprendizagem que permite a aquisição de outras aprendizagens.

É muito importante conceituar a leitura como uma fonte de informação, aprendizagem, que permite resolver problemas, tanto contexto escolar e na vida diária, tornando possível para expandir a visão de mundo, o desenvolvimento da sensibilidade e processos intelectuais, a partir deste perspectiva, esta pesquisa é importante porque torna possível sintetizar novos conhecimentos a partir de relatórios anteriores; da mesma forma, por isso eles se conheceram e entendido conhecimentos produzidos em diferentes épocas e em diferentes campos.

O objetivo é determinar o efeito de compreensão de leitura no desenvolvimento intelectual das crianças na quinta série na Geral da Educação Básica Middle School em 28 de maio período Canuto Parish Chone Canton 2014-2015.

Palavras-chave: Compressão leitura; intelectual; profesores; alunos e pais. 


\section{Introducción}

En el planteamiento del problema se analizan los tres contextos: macro, meso, micro, en los países latinoamericanos muchas personas no saben leer, se ha comprobado que tiene muchas falencias para entender y comprender lo que leen. Algunas investigaciones en el campo de la lectura, han demostrado que las experiencias del niño influyen directamente en la interpretación y comprensión de cualquier trabajo de lectura; en la medida en que haya mayor imaginación, la búsqueda del significado y la reconstrucción del mensaje del autor por parte del lector, fue fecundo; así también en nuestro país Ecuador estas limitaciones o carencias que presentan los escolares con respecto a la comprensión lectora se hace más evidente a medida que avanza el sistema educativo.

En el contexto micro, en la Unidad Educativa 28 de Mayo de la ciudad de Chone, parroquia de Canuto, se ha evidenciado la deficiente aplicación de la lectura comprensiva y su influencia en el desarrollo intelectual, puesto que los y las docentes no aplican lectura comprensiva en el desarrollo de las clases, no se fomenta la práctica de leer en los estudiantes de básica media específicamente de quinto grado, la aplicación e innovación de métodos y técnicas para mejorar la lectura comprensiva en concordancia con el desarrollo intelectual ya que ayuda a su desarrollo integral de todos ellos, y así las evaluaciones son mejoradas y apoyan a la comprensión de la lectura.

En la justificación este trabajo de titulación cobra importancia porque se evidencia que la lectura es una fuente de información, de aprendizaje, que permite resolver problemas tanto del contexto escolar como de la vida cotidiana, posibilitando ampliar la visión del mundo, el desarrollo de la sensibilidad y los procesos intelectuales y por eso el objetivo general responde a determinar la importancia de la lectura comprensiva en el desarrollo intelectual de los niños y niñas de quinto 
grado en la Educación General Básica Media, de la parroquia de Canuto, cantón Chone en el año lectivo 2014.

\section{Materiales y métodos}

\section{Tipo de investigación.}

Investigación Diagnóstica, se pudo realizar un diagnóstico de la lectura comprensiva en el desarrollo intelectual de los niños y niñas.

Investigación bibliográfica, se utilizó para la recopilación textual de la información con teorías relacionada a la lectura comprensiva y el desarrollo intelectual.

Investigación de campo, se la realizó mediante la recopilación de informantes. Información primaria: Encuestas, entrevistas y guía de observación.

\section{Nivel de investigación}

Los niveles de investigación en que se apoyó fue el nivel explorativo, porque se auscultó sobre la lectura comprensiva y el desarrollo intelectual y el nivel descriptivo ya que se detalló todo lo explorado.

\section{Métodos}

En el presente trabajo de titulación se utilizó los siguientes métodos:

Con el método de inducción, se la utilizó en el desarrollo del diagnóstico de la lectura comprensiva y el desarrollo intelectual por parte de los docentes y realizar generalizaciones a partir de los resultados obtenidos, es decir de las conclusiones. 
Con el método de la deducción, se empleó para analizar procedimientos de la lectura comprensiva y el desarrollo intelectual dentro del aula de clases.

A través del método analítico se pudo obtener información básica, se analizó el problema y su fundamentó científicamente; y la síntesis se utilizó sobre la base de los resultados obtenidos previamente en el análisis. Posibilitando la sistematización del conocimiento.

Con el método sintético, se estableció la unión entre las partes previamente analizadas y así descubrir las relaciones esenciales y características generales entre ellas.

\section{Técnicas de recolección de información.}

Las técnicas que se aplicaron en el presente trabajo de titulación fueron: La entrevista a docentes y directivos, la guía de observación a los estudiantes y la encuesta a los padres para conocer el nivel de lectura comprensiva y su influencia en el desarrollo intelectual de los estudiantes.

\section{Población}

\begin{tabular}{|l|c|}
\hline \multicolumn{2}{|l|}{ Escuela de Educación Básica "28 de Mayo } \\
\hline ACTORES & NÚMERO \\
\hline Estudiantes & 30 \\
\hline Docentes & 1 \\
\hline Padres de Familias & 20 \\
\hline Directivos & 1 \\
\hline Total & $\mathbf{5 2}$ \\
\hline
\end{tabular}

La muestra del presente trabajo de titulación es del 100\% de los estudiantes, directivos, docentes, padres y madres de familias de la Escuela de Educación Básica 28 de Mayo. 


\section{Resultados.}

Comprobamos que la hipótesis: "La lectura comprensiva influye en el desarrollo intelectual de los niños y niñas en la Educación General Básica Media”, comprobamos que es verdadera, así lo indica la pregunta $\mathrm{N}^{\circ} 7$ de la encuesta realizada a los padres de familia; donde el gráfico \# 7 nos indica que el 50\% la lectura influye en el desarrollo intelectual de sus hijos.

En la pregunta $\mathrm{N}^{\circ} 7$ realizada a los padres de familia, sobre si consideran a la lectura como ayuda para el desarrollo intelectual, el gráfico \#7 nos demuestra que el 50\% expresaron que a veces la lectura les ayuda al desarrollo intelectual de su hijo, significa que la maestra debe motivar a sus estudiantes con técnicas activas para mejorar la parte intelectual.

De la entrevista realizada a la docente de quinto año básico en la pregunta $\mathrm{N}^{\circ} 6$ se le preguntó qué estrategias se deben implementar para generar un mejor desarrollo intelectual, manifiesta que los estudiantes deben escribir sus propios mitos, leyendas, cuentos, obras literarias donde puedan desarrollar su coeficiente intelectual.

En la pregunta \# 2 de la ficha de observación realizada a los estudiantes sobre si practican lectura comprensiva; el gráfico \# 10 nos indica que el 50\% a veces practican, por esta razón los resultados obtenidos determinan que si existe una deficiente aplicación de técnicas activas por parte de los docentes sobre lectura comprensiva en el desarrollo intelectual de los estudiantes de quinto año básico. 


\section{Conclusiones.}

Los estudiantes de la Unidad Educativa 28 de Mayo del cantón Chone se encuentran poco interesados en la lectura comprensiva, la cual la ven como una actividad rutinaria por la falta de las estrategias de la lectura comprensiva.

Los estudiantes no les agrada muchos las horas de lectura, no practican lectura comprensiva, no comprenden lo que leen y no les gusta preguntar cuando no entienden el significado de palabras desconocidas por eso tienen un bajo nivel en la lectura comprensiva.

Los docentes no trabajan con técnicas activas de lectura comprensiva, por lo tanto los estudiantes no están estimulados y de esta manera falta el interés de la lectura en ellos y tienen un bajo nivel intelectual.

El limitado desarrollo intelectual obstaculiza estrategias de gran impacto positivo como aprendizaje colaborativo, círculos de aprendizaje, pensamiento sistémico.

La falta de capacitación en los docentes de la escuela 28 de Mayo de la parroquia de Canuto del cantón Chone sobre los temas estrategias, técnicas de lectura comprensiva, hace que los estudiantes no tenga interés en ella.

\section{Recomendaciones}

Que se apliquen técnicas y estrategias activas en el proceso de la lectura comprensiva para que mejoren el desarrollo intelectual de los estudiantes.

Que se desarrollen en el proceso de la lectura temas de cotidianidad para que despierten en ellos el interés y puedan comprender, reflexionar, redactar y emitir mensajes sobre lo leído. 
Viviana P. Patiño-Zambrano; Alexandra M. Alvarado-Álvarez; María D. Zambrano-Mendoza; José A. Barberan-Zambrano

Que se implementen en el proceso de la lectura estrategias didácticas que mejoren la comprensión lectora en las diferentes actividades individuales y grupales.

Que se generen espacios para la interacción grupal con visión compartida y a partir de un liderazgo participativo y así se potencialice el aprendizaje cooperativo como una estrategia de desarrollo intelectual.

A los docentes que se capaciten a través de talleres para conocer y aplicar las diferentes estrategias y tipos de lectura que ayuden a lograr un alto nivel de desarrollo intelectual en los estudiantes.

\section{Bibliografía}

Arias, Juan de Dios, (2003), Problemas De Aprendizaje, Universidad Pedagógica Nacional, Bogotá DC Colombia

Agencia EFE (2001) Investigadores chinos descubren beneficios de educación de bebés, Pekín

Carrillo, A. (2006). Técnicas para motivar la lectura.

Cavallo, Guglielmo, (2001), Historia de la lectura en el mundo occidental, Taurus, Madrid.

Cuetos, F. (1996), Psicología de la lectura, diagnóstico y tratamiento de los trastornos de lectura, Madrid Escuela Española.

Estalayo, V, (2003), Leer Bien al alcance de todos, Biblioteca Nueva, Madrid.

Freire, Heredara Manuel, (2001), Separata Técnica de Lenguaje - $8^{\circ}, 9^{\circ}$ y 10mo Año de Educación General Básica, Ministerio de Educación. Edipcentro, Ecuador.

Garay, Estrategias de comprensión lectora. http://www.centroandino.org.

Hernández, Días Fabio (1996), Metodología del Estudio, Santa fe de Bogotá DC, Mc Graw Hill

Hidalgo, Matos Menigno, (2007), Materiales Educativos, INADEP, Tercera Edición.

Izquierdo, Arellano Enrique, (2004), Didáctica y Aprendizaje grupal, Gráficas Lizette, Loja - Ecuador.

Manguel, Alberto, (1999), Una historia de la lectura, Grupo Editorial Norma, Bogotá. 
Millares, Agustín, (1995), Introducción a la Historia del Libro y de las Bibliotecas, Fondo de Cultura Económica, México.

\section{Webgrafía}

$\underline{\text { www.educación.gob.ec }}$

$\underline{\text { www.educarecuador.gob.ec }}$

http://www.inec.gob.ec/documentos varios/presentacion habitos.pdf 